\section{Lymphoproliferation, immunodeficiency and early-onset inflammatory bowel disease associated with a novel mutation in Caspase 8}

Caspase- 8 is a member of the aspartate-specific cysteine protease family that is typically synthetized as an inactive zymogen and activated upon an appropriate stimulus. Caspase- 8 plays an essential role in apoptotic signal transduction from the death receptor. Recruitment of procaspase- 8 into the death signaling complex leads to its dimerization, autoproteolytic cleavage and formation of a highly active heterotetramer. Caspase- 8 subsequently activates caspase- 3 , thereby initiating the proteolytic pathway, and ultimately resulting in the apoptotic disassembly of the cell. ${ }^{1}$ Additionally, caspase- 8 is also essential for various immune processes, such as lymphocyte activation, inflammasome regulation and cytokine production. ${ }^{2,3}$

Only one disease-causing mutation in the CASP8 gene in humans has been described thus far. Originally, NM_001228:c.793C>T, p.R265W was found in a homozygous constitution in two children of consanguineous parents with clinical manifestations resembling autoimmune lymphoproliferative syndrome (ALPS) and immunodeficiency, characterized by recurrent infections and a poor response to immunization. ${ }^{4}$ Later, the same mutation was found in other patients who were distantly related to the first two cases and presented with adult onset end organ lymphocytic infiltration and granulomas, with clinical features of immune deficiency and dysregulation. ${ }^{5}$ Here, we present a novel disease-causing mutation in the human CASP8 gene.
Our patient is a 15 -year-old boy who presented at the age of 5 months with failure to thrive, lymphadenopathy, recurrent enterorrhagia, rectal prolapse, rectal polyps and perianal fistula, hepatosplenomegaly and anemia. $\mathrm{He}$ underwent an appendectomy at 4 years for persistent abdominal pain, revealing severe mesenteric lymphadenopathy. Subsequently, he suffered from recurrent sinopulmonary infections, otitis and fevers of unknown origin. At 5 years of age, he developed pneumonia, during which a chronic atelectasis of the left lung lobe and generalized lymphadenopathy were noted on computed tomography scan. The patient also suffered numerous EBV infections/reactivations. Bone marrow samples displayed signs of chronic cellular activation but no malignancy was found (Online Supplementary Figure S1). Laboratory examination showed mild dysgammaglobulinemia (IgG 10.3 g/1, norm 7.31 - 12.75 g/l; IgA 0.78 g/l, norm 0.91 - $1.7 \mathrm{~g} / \mathrm{l}$; IgM $0.18 \mathrm{~g} / \mathrm{l}$, norm 0.47 - $1.8 \mathrm{~g} / \mathrm{l}$ ) and low specific antibody response to polysaccharide vaccines (anti-HiB $0.37 \mathrm{mg} / \mathrm{l}$, norm $>1 \mathrm{mg} / \mathrm{l}$, anti-pneumococcus $3.63 \mathrm{mg} / \mathrm{l}$, norm $>6 \mathrm{mg} / \mathrm{l})$. Immunophenotyping of peripheral blood cells revealed increased activated $\mathrm{T}$ cells with a maturation shift towards effector memory cells $\left(70.3 \%\right.$ in $\mathrm{CD}^{+}$, norm $0.49-25 \%$ (884 cells/ $\mu \mathrm{l}$, norm $5.1-210$ cells $/ \mu \mathrm{l})$ and $72.1 \%$ in $\mathrm{CD}^{+}$, norm 4 $100 \%$ (1541 cells/ $\mu \mathrm{l}$, norm 16 - 810 cells/ $\mu \mathrm{l})$ ), and a low level of naïve cells. Accordingly, T-cell receptor excision circles (TREC) measured at the age of nine years were also low, forming only 660 copies per $1 \times 10^{6}$ cells which is below the level observed in children of the same age group. In contrast, B cells showed a decreased count of mature cells. Interestingly, double negative
A

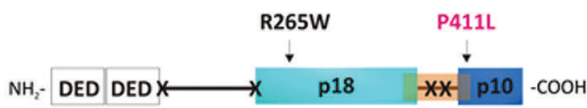

C

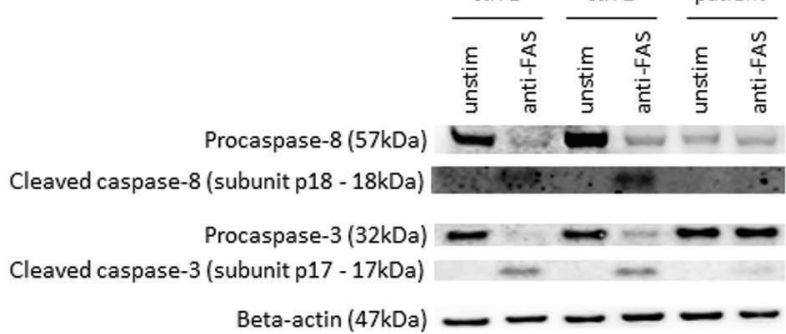

D

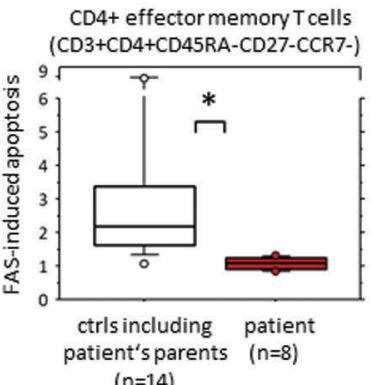

$(n=14)$

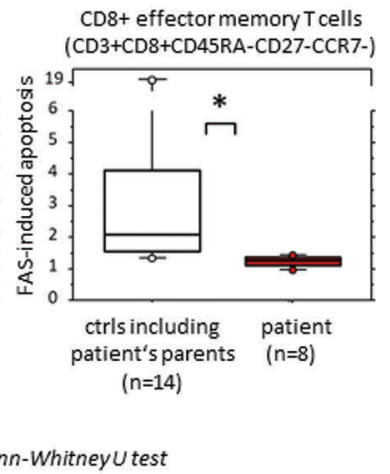

Figure 1. Procaspase- 8 structure and impaired caspase-8 expression and activity. Homozygous CASP8 mutation c.1232C $>$ T, p.P411L is located in the linker of the protein (A - scheme, B - NMR structure, PDB code $2 \mathrm{~K} 7 \mathrm{Z}^{6}$ ). DED, death-effector domain. Black crosses show cleavage resulting in activation of the enzyme. The c.1232C>T, p.P411L mutation impaired caspase-8 expression (C, row 1), as well as cleavage $(\mathrm{C}$, rows 1,2$)$ and activity $(\mathrm{C}$, rows 3,4$)$ upon anti-FAS stimulation. Apoptosis measured as Annexin $\mathrm{V}$ positivity in three independent experiments was also decreased (D). 
$\mathrm{CD}^{+}{ }^{+} \mathrm{TCRab}{ }^{+} \mathrm{CD} 4-\mathrm{CD} 8-\mathrm{T}$ cells were not increased in any of the measured timepoints (for more details, see Online Supplementary Table S1). The patient was regularly followed and treated symptomatically.

To exclude X-linked lymphoproliferative syndrome and activated PI3K-delta syndrome SH2D1A, BIRC4, $P I K 3 C D$ and PIK3R1 genes were analyzed by Sanger sequencing initially. Upon negative results, whole-exome sequencing was performed using the NextSeq Illumina platform. Variant calling, data analysis and annotation were carried out as described in Online Supplementary Methods. Multiple variant filtering strategies were used to prioritize genetic variants based on population frequency, the patient's phenotype, mode of inheritance and variant severity as assessed by prediction tools. Using this protocol, a previously unpublished variant in the CASP8 gene, NM_001228:c.1232C>T, p.P411L, was detected in a homozygous trait. The variant was not found in dbSNP, the NHBLI Exome Sequencing Project, or the 1000 Genomes Project database, and it was predicted to be deleterious by most prediction tools (Online Supplementary Table S2). Sanger sequencing confirmed the homozygous variant in the patient and a heterozygous carrier status in the patient's parents and his brother, who are healthy (Online Supplementary Figure S2). Clinical manifestation in our patient, harboring the p.P411L mutation, is only partially concordant with two previously published p.R265W patients, where the gastrointestinal symptoms were not described with the exception of chronic diarrhoea in one patient. ${ }^{4}$ Veryearly-onset gastrointestinal involvement in our patient thus expands the array of potential phenotypical features of CASP8 mutations. Interestingly, while it dominated the clinical course in the first years of life, it later subsided to be replaced with severe respiratory infections, including pneumonias, and chronic lung damage. On the other hand, the clinical presentation of the other two previously reported adult patients with p.R265W mutation, such as adult-onset pulmonary hypertension necessitating a lung transplant and complex neurological disease, ${ }^{5}$ have been absent in our patient so far. We suggest that the specific genetic background and the nature of the novel p.P411L mutation along with other potential co-factors, such as infections, may account for the variant inter-individual clinical presentation. Online Supplementary Table S3 shows a comparison of clinical features of the CASP8 c. $1232 \mathrm{C}>\mathrm{T}$ patient with the previously published cases. ${ }^{4,5}$

While the originally described p.R265W mutation is located in the p18 subunit, the novel p.P411L mutation is positioned in the part of the linker that remains attached to the p10 subunit after self-cleavage by procaspase- 8 during caspase-8 activation (Figure 1A, 1B, Online Supplementary Figure S2). ${ }^{6,7}$ We hypothesized that the p.P411L variant may modify the arrangement of the linker and consequently affect the dimerization and proper cleavage, as seen in previous experiments with artificially manufactured p.T484D mutation located in the dimerization region. ${ }^{8}$ Indeed, stimulation via FAS receptor failed to induce cleavage of procaspase- 8 in the patient's cells which, together with an impaired cleavage of its substrate caspase- 3 , indicated decreased caspase- 8 function (Figure 1C). Moreover, reduced FAS-mediated apoptosis of the patient's effector memory T cells further confirmed the disruption of apoptotic pathway at a single cell level (Figure 1D). Furthermore, the expression of procaspase- 8 itself was diminished in the patient's cells (Figure 1C).

Procaspase- 8 has also been shown to be involved in non-apoptotic signaling downstream of the T-cell recep-
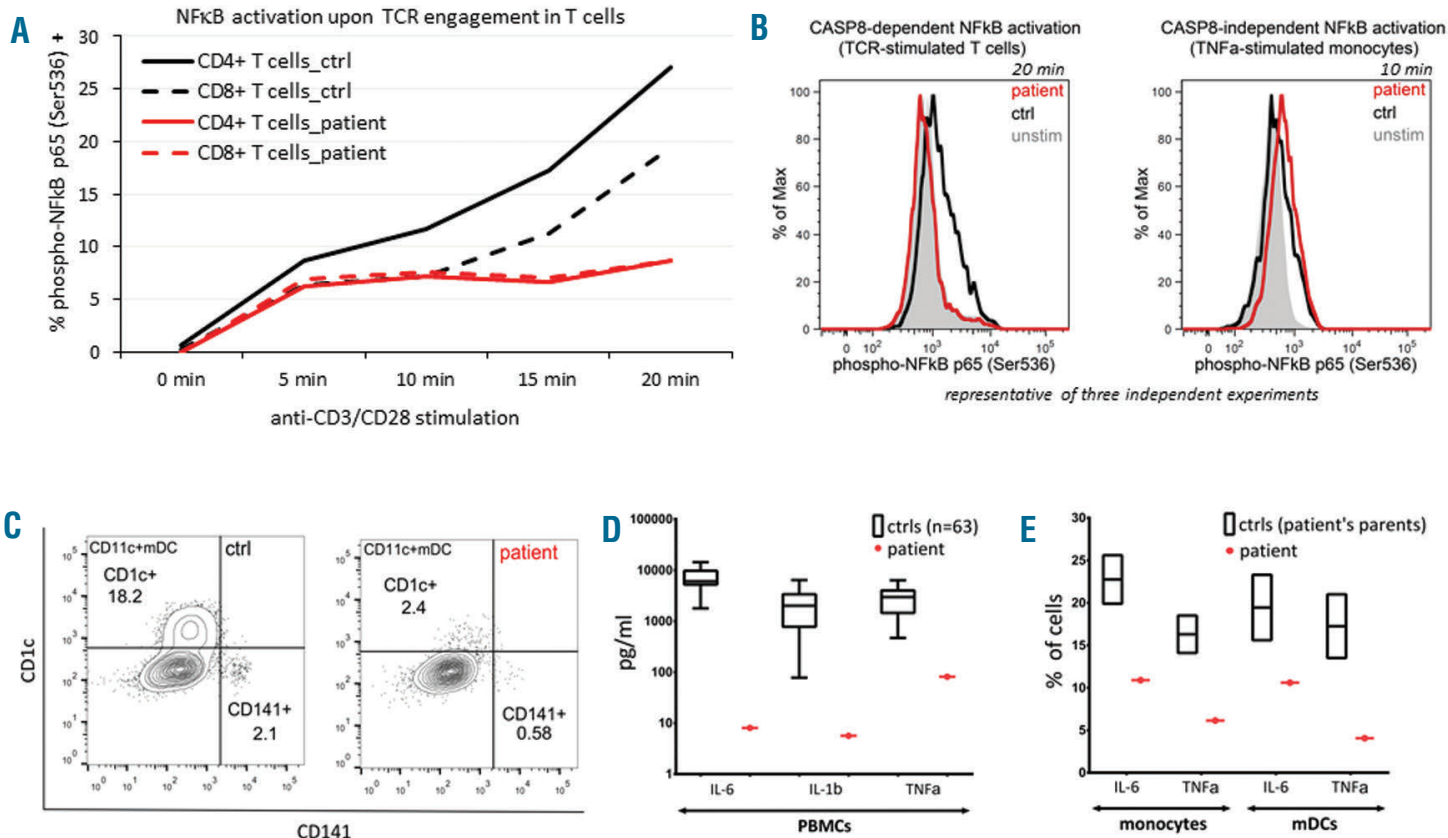

Figure 2. Alterations in T-cell signaling and TLR3-related innate cell characteristics. The patient's cells presented with impaired caspase-8-dependent NFKB activation (A, B) whereas caspase-8-independent NFKB activation remained unchanged (B). CD $1 \mathrm{c}^{+}$and $C D 141^{+} \mathrm{mDC}$ subpopulations were decreased in patient's PBMC (gated on Lin-HLA-DR+CD14-CD11 ${ }^{+}$cells. (C) IL-6, IL-1ß, and TNF $\alpha$ production induced by TLR3 agonist poly(I:C) measured in supernatant (D), and monocytes and $\mathrm{mDC}(\mathrm{E})$ was also diminished. 
tor (TCR) and consequently in signaling to NFKB. ${ }^{2}$ Timedependent NFKB activation was substantially decreased in the patient's T cells upon TCR engagement (Figure 2A). Importantly, no such reduction was observed after TNF $\alpha$ stimulation in monocytes, indicating that caspase8-independent NFKB activation ${ }^{2}$ was not affected (Figure 2B). Further evidence of disrupted non-apoptotic signaling was obtained by evaluation of T-cell activation (CD25, CD69) and proliferation (Ki-67, CellTrace Violet dye) which was substantially decreased in the patient's T cells, both naïve and effector memory, upon TCR stimulation, and only poorly restored by the addition of interleukin-2 (Online Supplementary Figure S3A, S3B.). In ALPS patients, lymphadenopathy is attributed to the accumulation of highly proliferative double negative $\mathrm{CD}^{+}{ }^{+} \mathrm{TCRab}^{+} \mathrm{CD}^{-}{ }^{-} \mathrm{CD} 8^{-} \mathrm{T}$ cells in the lymph nodes. ${ }^{9}$ In contrast, double negative $T$ cells were found to be unaltered in terms of count and proliferation in our patient (Online Supplementary Table S1 and Online Supplementary Figure S3C). Since the ability of the patient's T cells to activate and proliferate ex vivo was actually lower, despite their higher counts, we suggest that lymphadenopathy in caspase- 8 deficiency results from chronic activation and a gradual accumulation of the apoptosis-resistant $\mathrm{CD}^{+}$ and $\mathrm{CD} 8^{+} \mathrm{T}$ cells in affected lymph nodes. The role of the p.P411L mutation in naive T-cell lymphopenia is unclear. Since caspase- 8 signaling has been suggested to play a role in the downstream activation of signal transduction from immunoreceptors, ${ }^{4,10}$ poor generation and proliferation of the naïve T-cell compartment together with reduced memory $\mathrm{B}$-cell formation, either due to inadequate T-cell help or to intrinsic B-cell defects, might account for the clinical immunodeficiency observed.

Recurrent infections in the patients could also be promoted by diminished innate immune responses. Recent mounting evidence indicates the importance of caspase- 8 in Toll-like receptor (TLR)-mediated inflammation., ${ }^{3,11}$ Stimulation of murine caspase-8-deficient cells with a synthetic analog of double-stranded RNA (dsRNA) poly(I:C), which is a ligand of TLR3 and cytoplasmic receptors retinoid acid-inducible gene-I (RIG-I) and melanoma differentiation-associated gene 5 (MDA5), resulted in severe impairment of TNF $\alpha$ and IL- 6 production. ${ }^{12}$

In our patient, we demonstrated skewed myeloid dendritic cell ( $\mathrm{mDC}$ ) subpopulations, with a decreased number of both the more common $\mathrm{CD} 1 \mathrm{c}^{+}$and the very rare $\mathrm{CD} 141^{+} \mathrm{mDCs}$ (Figure 2C, Online Supplementary Figure S4), the only two DC subsets expressing TLR3. ${ }^{13}$ Stimulation via TLR3 led to impaired production of IL-6, IL-1 $\beta$, and TNF $\alpha$ in the patient's cells, both $\mathrm{mDCs}$ and monocytes (Figure 2D, 2E), while TLR4 engagement resulted in a similar level of produced cytokines as in healthy controls (Online Supplementary Figure S5).

In conclusion, we identified a novel CASP 8 mutation in the patient with lymphoproliferation, immunodeficiency and gastrointestinal involvement. Functional analyses of caspase-8-related pathways and cytokine production have supported a direct causal relationship between mutation p.P411L and a clinical phenotype. All laboratory and ex vivo functional tests were performed during a sufficiently long off-treatment period, avoiding the risk of treatment-biased results.

Veronika Kanderova, ${ }^{1}$ Hana Grombirikova, Irena Zentsova, ${ }^{3}$ Kamila Reblova, ${ }^{4}$ Adam Klocperk, ${ }^{3}$
Martina Fejtkova, ${ }^{1}$ Marketa Bloomfield, ${ }^{3}$ Barbora Ravcukova, ${ }^{2}$ Tomas Kalina, Tomas Freiberger ${ }^{2,4}$ and Anna Sediva ${ }^{3}$

TF and AS contributed equally to this work.

${ }^{1}$ Department of Pediatric Hematology and Oncology, $2^{\text {nd }}$ Faculty of Medicine, Charles University, and University Hospital Motol, Prague; ${ }^{2}$ Centre for Cardiovascular Surgery and Transplantation, Brno; ${ }^{3}$ Department of Immunology, $2^{\text {nd }}$ Faculty of Medicine, Charles University and University Hospital Motol, Prague and ${ }^{4}$ Central European Institute of Technology and Medical Faculty, Masaryk University, Brno, Czech Republic

Acknowledgments: we are especially grateful to the patient and his family for giving us consent to perform all research activities associated with the disease and to publish the results.

Funding: this work was supported by grants obtained from the Ministry of Health of the Czech Republic no. 15-28541A, no. 16-34414A, and no. NV18-05-00162, and a grant obtained from the Grant Agency of the Czech Republic no. P302/12/G101. Infrastructure was supported by the Ministry of Education, Youth and Sports NPU I LO1604 and institutional support obtained from University Hospital Motol 00064203 . We would like to acknowledge Dr. Erika Ondrusova from Pediatric Dept. of Faculty Hospital of Hradec Kralove for securing the pictures of bone marrow aspirates.

Correspondence: anna.sediva@fnmotol.cz doi:10.3324/haematol.2018.201673

Information on authorship, contributions, and financial \& other disclosures was provided by the authors and is available with the online version of this article at www. haematologica.org.

\section{References}

1. Tummers B, Green DR. Caspase-8: regulating life and death Immunol Rev. 2017(1);277:76-89.

2. Su H, Bidere N, Zheng L, et al. Requirement for Caspase-8 in NF-kB activation by antigen receptor. Science. 2005;307(5714):1465-1468.

3. Moen SH, Westhrin M, Zahoor M, et al. Caspase-8 regulates the expression of pro- and anti-inflammatory cytokines in human bone marrow-derived mesenchymal stromal cells. Immunity, Inflamm Dis. 2016;4(3):327-337

4. Chun HJ, Zheng L, Ahmad M, et al. Pleiotropic defects in lymphocyte activation caused by caspase- 8 mutation lead to human immunodeficiency. Nature. 2002;419(6905):395-399.

5. Niemela J, Kuehn HS, Kelly C, et al. Caspase-8 deficiency presenting as late-onset multi-organ lymphocytic infiltration with granulomas in two adult siblings. J Clin Immunol. 2015;35(4):348-355.

6. Keller N, Mares J, Zerbe O, Grütter MG. Structural and biochemical studies on Procaspase-8: new insights on initiator caspase activation. Structure. 2009;17(3):438-448.

7. Blanchard H, Donepudi M, Tschopp M, Kodandapani L, Wu JC, Grütter MG. Caspase-8 specificity probed at subsite S4: Crystal structure of the caspase-8-Z-DEVD-cho complex. J Mol Biol. 2000;302(1):9-16.

8. Boatright KM, Renatus M, Scott FL, et al. A unified model for apical caspase activation the cellular effects that distinguish apoptosis from other. Mol Cell. 2003;11(2):529-541.

9. Völkl S, Rensing-Ehl A, Allgäuer A, et al. Hyperactive mTOR pathway promotes lymphoproliferation and abnormal differentiation in autoimmune lymphoproliferative syndrome. Blood. 2016;128(2): 227-238.

10. Kennedy NI, Kataoka T, Tschopp J, Budd RC. Caspase activation is required for T cell proliferation. J Exp Med. 1999;190(12):1891-1895.

11. Feltham R, Vince JE, Lawlor KE. Caspase-8: not so silently deadly. Clin Transl Immunol. 2017;6(1):e124.

12. Takahashi K, Kawai T, Kumar H, Sato S, Yonehara S, Akira S. Cutting edge: roles of Caspase- 8 and Caspase-10 in innate immune responses to double-stranded RNA. J Immunol. 2006;176(8):4520-4524.

13. Piccioli D, Tavarini S, Borgogni E, et al. Functional specialization of human circulating CD16 and CD1c myeloid dendritic-cell subsets. Blood. 2007;109(12):5371-5379. 